\title{
Evolution of surgical techniques for a progressive risk reduction
}

\section{Bruno Amato1, Mario Santoro², Raffaele Izzo2, Giuseppe Servillo3, Rita Compagna1, Lorenza Di Domenico4, Veronica Di Nardo4, Giuseppe Giugliano5}

\author{
1 Department of Clinical Medicine and Surgery, University of Naples Federico II \\ 2 Department of Translational Medical Sciences, University of Naples Federico II \\ 3 Department of Neuroscience, University of Naples Federico II \\ 4 Department of Nuclear Physics and Radiation, Guglielmo Marconi University, Rome \\ 5 Department of Advanced Biomedical Sciences, University of Naples Federico II, Italy
}

\begin{abstract}
Advanced age is a strong predictor of high perioperative mortality in surgical patients and patients aged 75 years and older have an elevated surgical risk, much higher than that of younger patients. Progressive advances in surgical techniques now make it possible to treat high-risk surgical patients with minimally invasive procedures. Endovascular techniques have revolutionized the treatment of several vascular diseases, in particular carotid stenosis, aortic pathologies, and severely incapacitating intermittent claudication or critical limb ischemia. The main advantages of the endovascular approach are the low complication rate, high rate of technical success and a good clinical outcome. Biliary stenting has improved the clinical status of severely ill patients with bile duct stricture before major surgery, and represents a good palliative therapy in the case of malignant biliary obstruction.
\end{abstract}

\section{Introduction}

Advanced age is a strong predictor of high perioperative mortality, and patients over 75 years of age have a far higher surgical risk than younger patients. Progressive advances in surgical techniques now make it possible to treat high-risk surgical patients with minimally

Corresponding author: Bruno Amato, Department of Clinical Medicine and Surgery, University of Naples Federico II, Via S. Pansini 5, 80131 Napoli, Italy. E-mail: bruno.amato@unina.it

Key words: Evolution of surgical therapy; carotid artery stenting; drugseluting balloon; endovascular repair aortic dissection; geriatric population.

Received for publication: 27 November 2016

Accepted for publication: 7 December 2016

(C) Copyright B. Amato et al., 2017

Tipografia PI-ME Editrice, Italy

Monaldi Archives for Chest Disease 2017; 87:844

doi: 10.4081/monaldi.2017.844

This article is distributed under the terms of the Creative Commons Attribution Noncommercial License (by-nc 4.0) which permits any noncommercial use, distribution, and reproduction in any medium, provided the original author(s) and source are credited. invasive procedures. Endovascular techniques have revolutionized the treatment of several vascular diseases, in particular carotid stenosis, aortic pathologies, and severely incapacitating intermittent claudication or critical limb ischemia. The main advantages of the endovascular approach are the low complication rate, high rate of technical success and a good clinical outcome even in the long term. Biliary stenting has improved the clinical status of severely ill patients with bile duct stricture before major surgery, and represents a good palliative therapy in the case of malignant biliary obstruction.

\section{Percutaneous treatment of peripheral artery occlusive disease}

Percutaneous transluminal angioplasty (PTA) is a mini-invasive technique for the treatment of patients affected by severely incapacitating intermittent claudication or critical limb ischemia [1]. The main advantages of the endovascular approach are the low rate of complications, which range from 0.5 to $4 \%$, the high rate of technical success, estimated around $90 \%$ also for the treatment of long occlusions, and the good clinical outcome [2]. Currently, PTA is the therapy of choice for revascularization of the aortoiliac and femoropopliteal arteries and arteries of the leg: in fact in many centers, endovascular therapy is the therapeutic approach most frequently adopted [3]. However, it is not without complications, particularly the rate of incidence of restenosis is still unacceptably high. The main obstacle to the placement of first-generation nitinol metal stents is the marked intimal hyperplasia which is responsible for intrastent restenosis in $25 \%$ of cases at 12 months and in $50 \%$ at 24 months. In the course of recent years, drug-eluting balloons (DEBs) have been created, as well as new types of stent both metallic and medicated (drug-eluting stent, DES), covered stent grafts, and new systems of atherectomy to reduce the incidence of restenosis and improve the long term outcomes. The second-generation nitinol stents have a greater flexibility, particularly in the axial sense, and more resistance to fracture, characteristics which make these stents indicated also for the treatment of more complex lesions; the SUPERA SFA registry enrolled 107 patients with complex femoropopliteal lesions treated with insertion of a Supera stent, showing a patency rate of $76 \%$ at 24 months [4]. Today, PTA with a DEB, hence without stent placement, is becoming more and more common. The technique with DEB as opposed to PTA with stent placement allows a homogeneous release of the antiproliferative drug to the vessel wall without the need to leave foreign prosthetic material in situ which could trigger an inflammatory process, and a potentially shorter duration of the dual antiplatelet therapy. In addition, the DEB can be applied in 
anatomical zones which are sites of movement, such as the common femoral artery and popliteal artery, in which the placement of a stent could be subject to frequent complications due to fracture of the stent itself $[5,6]$. Nevertheless, there are limitations to the use of DEB, such as the incapacity to treat flaps of dissecations, and a lower efficacy in the case of severely calcified arteries on account of the smaller quantity of antiproliferative drug absorbed by the arterial wall.

\section{Stenting of the carotid artery}

Ischemic stroke is caused in 10-15\% of all cases by a stenosis of the internal carotid artery. In patients with carotid artery disease, the aim of revascularization is to prevent stroke. For about 50 years, carotid endoarterectomy (CEA) was the standard treatment for severe carotid stenosis. But, over the last 15 years, carotid artery stenting (CAS) has gained acceptance as an alternative to open surgery - this mini-invasive treatment was developed as a method of carotid revascularization in situations where CEA was technically difficult to perform or in patients at high surgical risk [7]. The comorbidities associated to a high risk of complications with endoarterectomy include: elderly age ( $>75$ years), chronic heart failure in NYHA class III or IV, unstable angina (CCS III/IV), chronic ischemic heart disease involving epicardial vessels with $>70 \%$ stenosis, recent acute myocardial infarction (AMI), severe aortic stenosis, ejection fraction $<30 \%$, severe COPD, and severe chronic kidney failure. Elderly patients thus constitute a patient group that can benefit from endovascular treatment $[8,9]$. In the CREST trial, in which 2502 patients were enrolled, one group underwent carotid stenting with the cerebral protection system and another group underwent CEA. During a mean follow-up of 2.5 years, no significant difference was observed in the incidence of primary endpoints (stroke, AMI, death for any cause during the periprocedural phase, ipsilateral stroke within 4 years after randomization into the study) between patients who underwent stenting and those who underwent CEA (7.2 and $6.8 \%$, respectively; HR $1.11 ; 95 \%$ CI $0.81-1.51 ; p=0.51$ ) [10]. Nevertheless, of note there was, in the second half of the study, a significant reduction in the rate of stroke in the group of patients who underwent stenting, suggesting a learning curve effect on the endovascular technique. A meta-analysis involving 3754 patients treated with stenting and 3723 patients who underwent CEA showed that, at 30 days from revascularization, the endovascular technique was significantly associated to a high risk of stroke and of mortality, while the incidence of AMI and of cranial nerve lesions was significantly higher in the group of patients who underwent open surgery, especially symptomatic patients and elderly patients; in addition, 30 days from revascularization, the efficacy of the two strategies in terms of prevention of homolateral ictus and their safety in terms of restenosis or need to repeat the revascularization was similar in all studies [11]. Recently, a double layer mesh stent has been developed with greater flexibility enabling it to adapt to an eventual tortuous path of the carotid artery while, at the same time, it consents an optimal cover of the plaque. The double layer mesh stent is characterized by an internal layer of mesh micromesh to cover the plaque and a self-expandable external layer in nitinol which guarantees the characteristic flexibility of the open-cell stents [12]. The size of the micromesh cell is extremely small $(0.381$ $\mathrm{mm}^{2}$ ) permitting an accurate cover of the plaque, rendering this technology safe and promising: in fact, at 30 days from revascularization this stent is associated with a low percentage of plaque prolapse and an optimal clinical outcome, but it remains to be seen if it will reduce the neurological events associated with the endovascular procedure at long-term follow-up.

\section{Aortic dissection}

Acute type A aortic dissection, i.e. with involvement of the ascending aorta - following Stanford's classification, is an urgent surgical intervention with an extremely high mortality rate, estimated between 28 and $58 \%$ of patients who are operated rapidly [13]. Advanced age is a strong predictor of high perioperative mortality: in fact, in 2001 , the rate of perioperative mortality in patients in their 80 s was reported to be $83 \%$ [14]; however, in the last few years, thanks to technological advances and greater experience of surgeons in handling this disease, the rate has dropped to $13-25 \%$, accompanied though by an increase in the number of patients surviving who present severely invalidating post-treatment outcomes. The IRAD study has demonstrated that, despite the significant increase in the rate of surgical mortality with advancing age, surgical treatment of patients aged $<80$ years is associated to significantly lower rates of in-hospital mortality than medical therapy, and surgery can reduce the in-hospital mortality also in patients in their 80 s affected by type A aortic dissection; thus, surgical treatment could be considered in all patients with type A aortic dissection independently of their age. For patients with dissection of the thoracic aorta without involvement of the ascending aorta (Stanford type B dissection), endovascular treatment through insertion of an endoprosthesis, as an alternative to open surgery, is an extremely interesting therapeutic innovation due to its reduced invasiveness, enabling the treatment also of patients at high surgical risk, and thus representing a life-saving therapeutic option that can prevent complications such as aortic rupture and malperfusion syndrome [13]. The endoprosthesis functions through a skeleton of nitinol which exerts a radial force and has properties of elasticity, thermal memory and extreme robustness. Hemodynamically stable patients are usually treated conservatively with medical therapy (survival rate at 1 year $\geq 80 \%$ ), but the long-term results are disappointing due to the possible expansion of the aneurysm and a cumulative mortality of $30 \%$ at 5 years. The therapeutic rationale for insertion of an endoprosthesis is to promote thrombosis of the false lumen provoking the occlusion and, thus, reestablish the normal anatomy of the aorta. With respect to open surgery, a lower incidence of mortality and morbidity is reported, particularly for complications related to medullary ischemia.

\section{Biliary stenting}

Biliary stenting is a procedure consisting of the insertion of a tubular prosthesis to eliminate a constriction of the biliary duct wall and prevent their reformation. The indications for this procedure are biliary duct obstruction caused by neoplasms of the pancreas, gallbladder, biliary ducts, liver or colon, damage to the biliary ducts arising following removal of the gallbladder or following a pancreatitis, primitive sclerosing colangitis, gallstones, radiotherapy, or abdominal injuries. The procedure can thus be performed to improve patients' clinical status before major surgery or as definitive palliative therapy in the case of malignant biliary obstruction. The prosthesis can be positioned with the aid of endoscopic retrograde cholangiopancreatography (ERCP) or percutaneous transhepatic cholangiography (PTC) [15]. In the first case, before inserting the stent, a sphincterectomy is performed which allows direct access to the interior of the biliary ducts; in some cases, however, before inserting the stent the obstructed biliary duct is expanded by inflating a balloon in its interior. PTC, on the other hand, involves needle puncture of the biliary ducts through the skin at the level of the right hip or epigastric region, to provide access 
to the duct for successive positioning of the stent in the biliary site. This treatment reestablishes normal patency of the biliary duct in $90 \%$ of cases. However, the procedure is not without complications, such as occlusion or dislocation of the stent, infections, cholecystitis, hemorrhage, pancreatitis, cholangitis, and intestinal lesions in the case of ERCP.

\section{Discussion}

Advances in surgical techniques have made it possible to treat patients at high surgical risk with increasingly less-invasive procedures. Endovascular surgery has revolutionized the therapeutic approach to many steno-obstructive vascular diseases due to its advantages of a low rate of complications, elevated percentage of technical success and good clinical outcomes also in the long term. However, the endovascular approach is still today limited by a high rate of restenosis, the main underlying pathophysiological cause of which is myointimal hyperplasia, triggered by inflammatory-immunological processes. For decades, carotid endoarterectomy represented the standard treatment for severe carotid stenosis; today, stenting of the carotid artery is considered a mini-invasive alternative to open surgery, which enables revascularization even in cases where endoarterectomy is technically difficult or in patients at high surgical risk. Thoracic aortic dissection is a dramatic event, aggravated by an extremely high rate of mortality. For patients with thoracic aortic dissection without involvement of the ascending aorta (Stanford type B aortic dissection) the insertion of an endoprosthesis, as an alternative to open surgery, constitutes a therapeutic technique with reduced invasiveness that permits the treatment also of patients at high surgical risk, with the aim to close the intimal tear, interrupt the blood flow in the false lumen and promote thrombosis of the latter. Also, the treatments of endoscopic surgery of the biliary ways and biliary stenting have improved the clinical status of patients with occlusions or stricture of the biliary ducts, representing a non-obstructive treatment alternative to open surgery in the case of biliary stones, or a temporary or definitive palliative treatment in the case of malignant biliary obstruction.

\section{Conclusions}

Advanced age is a predictor of high perioperative mortality in surgical patients. Advances in surgical techniques have made it possible to treat patients at high surgical risk through procedures which are increasingly less invasive. In particular, endoscopic surgery of the biliary ducts and endovascular treatments have revolutionized in recent years the therapeutic approach to many steno-obstructive biliary and vascular diseases; nevertheless, they are marked by a consistent rate of complications and relapse, which represents the current challenge for clinical research.

\section{References}

1. Schillinger M, Minar E. Percutaneous treatment of peripheral artery disease: novel techniques. Circulation 2012;126:2433-40.

2. Giugliano G, Perrino C, Schiano V, et al. Endovascular treatment of lower extremity arteries is associated with an improved outcome in diabetic patients affected by intermittent claudication. BMC Surg 2012;12(Suppl 1):S19.

3. Giugliano G, of Serafino L, Perrino C, et al. Effects of successful percutaneous lower extremity revascularization on cardiovascular outcome in patients with peripheral arterial disease. Int J Cardiol 2013;167:2566-71.

4. Scheinert D, Grummt L, Piorkowski M, et al. A novel self-expanding interwoven nitinol stent for complex femoropopliteal lesions: 24month results of the SUPERA SFA Registry. J Endovasc Ther 2011;18:745-52.

5. Stabile E, Magliulo F, Zhelev D, et al. Interim analysis at 6months from the LEG-flow Drug Eluting Balloon for the treatment of femoropopliteal occlusions (LEG-DEB) registry. Int J Cardiol 2016; 223:654-5.

6. Stabile E, Niglio T, Giugliano G, et al. Compression of a woven selfexpanding femoropopliteal stent leading to occlusion. JACC Cardiovasc Interv 2016;9:131-2.

7. Stabile E, Sannino A, Schiattarella GG, et al. Cerebral embolic lesions detected with diffusion-weighted magnetic resonance imaging following carotid artery stenting: a meta-analysis of 8 studies comparing filter cerebral protection and proximal balloon occlusion. JACC Cardiovasc Interv 2014;7:1177-83.

8. Quarto G, Benassai G, Gallinoro E, et al. Is surgery always the best treatment for severe carotid stenosis in the frail elderly? Ann Ital Chir 2015;86:9-12.

9. Amato B, Markabaoui AK, Piscitelli V, et al. Carotid endarterectomy under local anesthesia in elderly: is it worthwhile? Acta Biomed 2005;76 (Suppl 1):64-8.

10. Brott TG, HobsonRW II, Howard G, et al. Stenting versus endarterectomy for treatment of carotid-artery stenosis. N Engl J Med 2010; 363:11-23.

11. Economopoulos KP, Sergentanis TN, Tsivgoulis G, et al. Carotid artery stenting versus carotid endarterectomy: a comprehensive meta-analysis of short-term and long-term outcomes. Stroke 2011;42:687-92.

12. Stabile E, Giugliano G, Cremonesi A, et al. Impact on outcome of different types of carotid stent: results from the European Registry of Carotid Artery Stenting. EuroIntervention 2016;12:265-70.

13. Giugliano G, Spadera L, De Laurentis M, Brevetti G. Chronic aortic dissection: still a challenge. Acta Cardiol 2009;64 653-63.

14. Chiu P, Miller DC. Evolution of surgical therapy for Stanford acute type A aortic dissection. Ann Cardiothorac Surg 2016;5:275-95.

15. Dumonceau JM, Tringali A, Blero D, et al. Biliary stenting: indications, choice of stents and results: European Society of Gastrointestinal Endoscopy (ESGE) clinical guideline. Endoscopy 2012;44:277-98. 